CVIA

CASE REPORT

pISSN 2508-707X / eISSN 2508-7088 https://doi.org/10.22468/cvia.2016.00031 CVIA 2017;1 (1):86-88

CrossMark \& click for updates

Received: September 4, 2016

Revised: November 4, 2016

Accepted: November 17, 2016

Corresponding author

Ming-Yen Ng, MD

Department of Diagnostic Radiology,

The University of Hong Kong,

Queen Mary Hospital,

102 Pokfulam Road, Hong Kong, China

Tel: 852-2255-4524

Fax: 852-2855-1652

E-mail: myng2@hku.hk

\section{Aortic Coarctation Following Aortic Valve Replacement: Problem Solving with Multimodality Cardiac Imaging}

\author{
Ming-Yen Ng ${ }^{1,2}$, Qin Li', Lee Benson ${ }^{1,3}$, Eric Horlick', Rachel Wald' \\ 'Department of Cardiology, Toronto General Hospital, University Health Network, Toronto, Canada \\ 2Department of Diagnostic Radiology, The University of Hong Kong, Queen Mary Hospital, Hong \\ Kong, China \\ ${ }^{3}$ Department of Cardiology, The Hospital for Sick Children, Toronto, Canada
}

\begin{abstract}
We present a unique case of severe aortic narrowing identified in adulthood, six years after aortic valve replacement for aortic insufficiency, due to bicuspid aortic valve disease. We illustrate the importance of multimodality cardiac imaging in the diagnosis and treatment of this condition using echocardiography, cardiac CT, MRI, and angiography.
\end{abstract}

Key words Aortic coarctation - Aortic diseases, interrupted · Bicuspid aortic valve
Post-valvular replacement.

\section{INTRODUCTION}

We describe a case of a 33-year-old male that had an aortic valve repair for a bicuspid aortic valve with severe aortic regurgitation 7 years previously. CT and MRI demonstrated a severe narrowing at the aortic isthmus with no discernible connection. Catheter angiogram also demonstrated a nonvisible connection between the aortic arch and descending aorta. Based on the previous history of a bicuspid aortic valve, severe aortic coarctation was more likely than an interrupted aortic arch. A balloon expandable stent was inserted with satisfactory results.

\section{CASE REPORT}

A 33-year-old man presented to our institution with a history of mechanical aortic valve replacement (On-X \#23) for a bicuspid aortic valve with severe aortic regurgitation 7 years previously. He had intractable hypertension that required treatment with four distinct antihypertensive medications. He was referred to a community cardiologist who noted absent leg pulses and an initial CT showed severe aortic coarctation. He was subsequently referred to our centre. On initial examination, his right arm blood pressure was 154/90 $\mathrm{mm} \mathrm{Hg}$ and the pulses in his legs were absent. Heart sounds showed a normal S1, mechanical S2, and a grade $2 / 6$ systolic ejection murmur along with a left sternal

(c) This is an Open Access article distributed under the terms of the Creative Commons Attribution Non-Commercial License (http://creativecommons.org/licenses/by$\mathrm{nc} / 3.0$ ) which permits unrestricted non-commercial use, distribution, and reproduction in any medium, provided the original work is properly cited. border. There was no diastolic murmur.

An echocardiogram demonstrated normal function of the mechanical aortic valve and evidence of mild concentric left ventricular hypertrophy. Monophasic wave form, blunted systolic upstroke and antegrade diastolic flow were noted in the abdominal aorta Doppler (Supplementary Fig. 1A in the online-only Data Supplement). Chest X-ray showed bilateral inferior rib notching (Supplementary Fig. 1B in the online-only Data Supplement). Cardiac CT (Fig. 1A and B) and MR angiograms (MRA) (Fig. 1C and D) did not show a connection between the aortic arch and descending thoracic aorta. MRA demonstrated reconstruction of the descending aorta by collateral vessels, which further confirmed the anatomical assessment. The proximal descending thoracic aorta was dilated, which indicated post-stenotic dilatation associated with coarctation of the aorta. Based on this, we hypothesized that the preexisting short segmental coarctation had become atretic rather than an interrupted aortic arch. The lack of associated ventricular septal defect or patent ductus arteriosus seen frequently in patients with interrupted aorta also suggested this was probably an atretic coarctation. After multi-disciplinary discussion, intervention was thought to likely help provide the answer and to treat the condition. During catheterisation, the initial attempt, using an extra stiff exchange Guidewire as a straight wire to pass the narrowing, was unsuccessful. Subsequently, the back end of an 0.035 wire was then used to punch through the occlusion into the descending aorta. This wire was removed and an extra stiff exchange length wire was advanced. A 14-French Mullins 

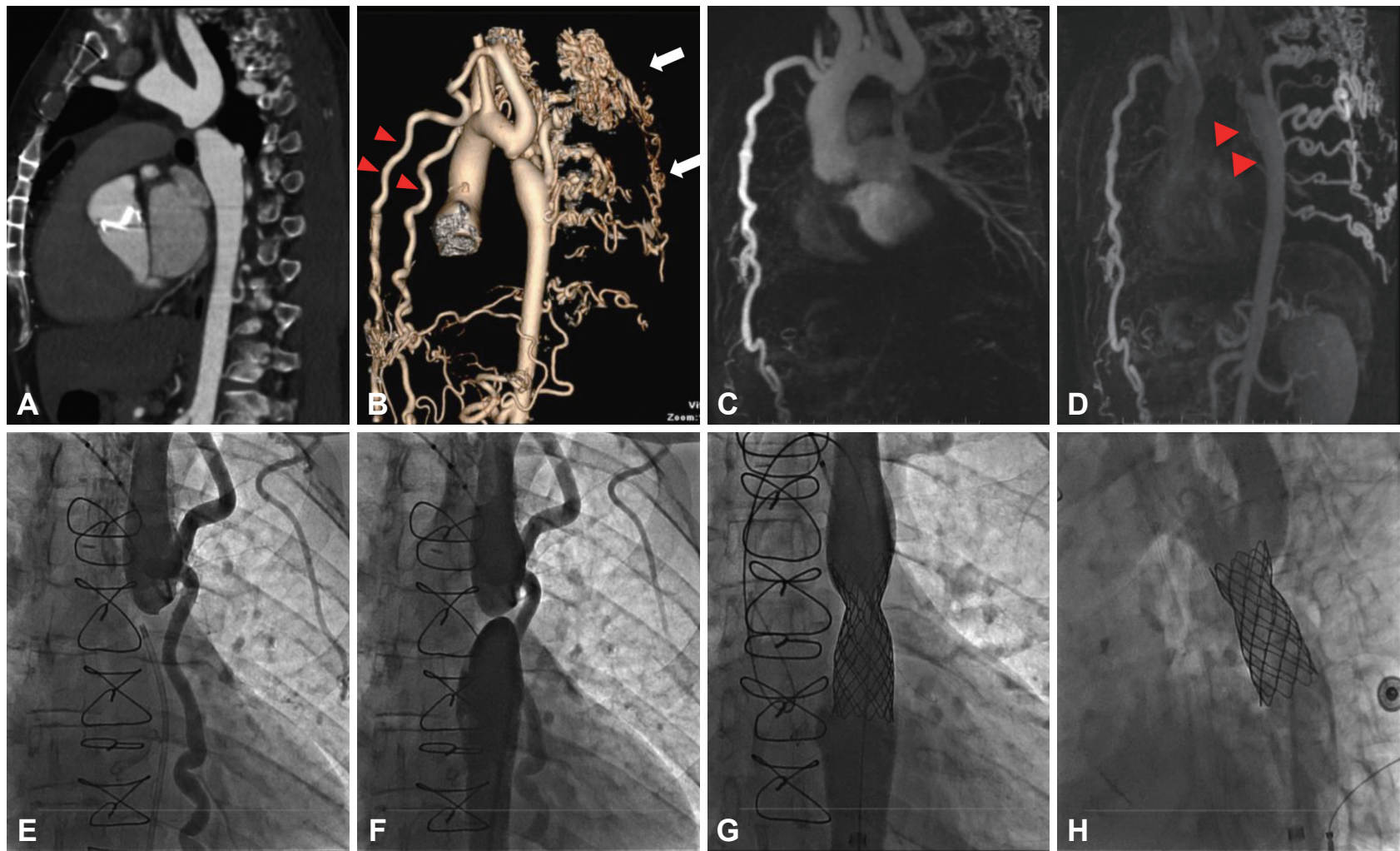

Fig. 1. CT angiogram (A) demonstrates severe aortic coarctation just distal to the left subclavian artery and a mechanical valve in a good position. The volume rendered CT (B) shows the plethora of large collateral vessels which supply the descending thoracic aorta. Note the prominent left and right internal mammary arteries (red arrowheads) and intercostal collaterals (white arrows). Early phase MRA (C) shows contrast filling of the ascending aorta, arch proximal to the site of atresia, as well as collateral vessels. Note the absence of contrast distal to the occlusion. Later phase MRA (D) demonstrates subsequent filling of the descending aorta (arrowheads) by collaterals. Angiogram with a catheter in the aortic arch (E) shows filling of the aortic arch and the collateral vessels, including the left internal mammary artery. Note non-filling of the descending thoracic aorta, suggestive of atresia at the aortic isthmus. Angiogram using two catheters $(F)$ in both the aortic arch and descending thoracic aorta clearly demonstrates the focal occlusion at the aortic isthmus. (G) Successful advancement of a wire past the site of occlusion and subsequent stenting. $(\mathrm{H})$ Final angiogram after successful stenting. MRA: MR angiograms.

sheath was advanced to cross the occlusion with no resistance. A $55 \mathrm{~mm}$ Cheatham-Platinum covered stent on a $22 \mathrm{~mm}$ balloon (based on measurements made with the approximate size of the aorta being $22 \mathrm{~mm}$ ) was advanced into position and inflated twice. Angiography confirmed good position of the stent with no evidence of contrast extravasation (Fig. $1 \mathrm{G}$ and $\mathrm{H}$ ) and only a $3 \mathrm{~mm}$ pressure gradient across the stent. Post-stenting, the patient became normotensive and the majority of the patient's medications were discontinued.

\section{DISCUSSION}

Aortic coarctation is an important congenital lesion associated with a bicuspid aortic valve. If left untreated, it may result in severe uncontrolled hypertension, left ventricular remodeling, and heart failure. For our patient, this diagnosis was missed prior to aortic valve replacement in his native country. Utilization of covered stents for percutaneous treatment of aortic coarctation, atretic coarctation, and even an interrupted aorta has been shown to be successful in both adult and pediatric populations [1-4]. As shown in our case, intervention can help confirm a diagnosis and offer definitive treatment if a diagnosis of aortic coarctation, atretic coarctation or interrupted aortic arch are difficult to distinguish. However, to the best of our knowledge there are currently no publications which suggest predictors of successful recanalisation, which is likely due to the rarity of this condition. In addition, cardiac $\mathrm{CT}$ and MRI provide high quality images which can be used to guide an interventional cardiologist during percutaneous treatment of these lesions.

\section{Supplementary Materials}

The online-only Data Supplement is available with this article at https:// doi.org/10.22468/cvia.2016.00031.

\section{Conflicts of Interest}

The authors declare that they have no conflict of interest.

\section{REFERENCES}

1. Pedra CA, Fontes VF, Esteves CA, Arrieta SR, Braga SL, Justino H, et al. Use of covered stents in the management of coarctation of the aorta. Pediatr Cardiol 2005;26:431-439.

2. Tzifa A, Ewert P, Brzezinska-Rajszys G, Peters B, Zubrzycka M, Rosenthal 
E, et al. Covered Cheatham-platinum stents for aortic coarctation: early and intermediate-term results. J Am Coll Cardiol 2006;47:1457-1463.

3. Freund MW, Vollebregt AM, Krings G, Vonken EP, Agostoni P, Meijboom FJ. Native atretic coarctation of the aorta in a 37 -year-old hypertensive woman, treated with a low-profile covered stent. Neth Heart J 2013;21:206207.

4. Goel PK, Moorthy N. Percutaneous reconstruction of interrupted aortic arch in an adult. JACC Cardiovasc Interv 2013;6:e21-e22. 